\title{
Postmarketing drug surveillance by record linkage in Tayside
}

\author{
IAIN K CROMBIE, SHEILA V BROWN, AND JOHN G HAMLEY \\ From the Medicines Evaluation and Monitoring Group, Ninewells Hospital and Medical School, Dundee DDI \\ 9SY, UK
}

SUMMARY The feasibility of conducting postmarketing drug surveillance by record linkage in Tayside was assessed. The key feature of the method is that all hospital discharge data are already computerised by the area health board and may be accessed through the unique community health number (CHNo) which has been allocated to all Tayside residents. The 12861 prescriptions for cimetidine dispensed in Tayside over a nine month period were collected and the CHNo identified for $76 \%$. These corresponded to 3802 individuals and their discharge data, together with those for an equal number of controls matched by age, sex, and general practitioner were retrieved from the computer. The expected excess of those diseases for which cimetidine is prescribed (peptic ulcer and oesophagitis) was observed. Other drug-disease associations were observed but may have been due to confounding and emphasise the inadequacy of community based controls. The major advantages of record linkage are, firstly, the low cost of the method, the present study costing just over $£ 12000$, and, secondly, the duration of patient follow up which may may be extended for as many years as required simply by rerunning the computer programs. To assemble large patient cohorts the study would have to be extended to other area health boards that are currently developing similar computer systems. Record linkage may provide a cost effective method for the follow up of patients to identify serious adverse drug reactions, particularly those that take several years to develop.

The Tayside Record Linkage Study was established after an initiative by the adverse reaction subcommittee of the Committee on Safety of Medicines. The object was to investigate the feasibility of linking hospital medical records to family doctor prescribing for the whole of Tayside. The potential of record linkage for the detection of adverse drug reactions had been clearly shown by Skegg and Doll in the Oxford Community Record Linkage Project. ${ }^{1}$

The attraction of the Tayside area for this type of study is that all the patients are identified by a unique community health number, and this number is used on all computerised records of hospital inpatient morbidity statistics and outpatient contacts. ${ }^{2}$ The scheme currently embraces some 400000 people, but similar systems are being established in two other health board areas (Argyll and Clyde, and Grampian) so that it could potentially be extended to cover $1 \cdot 2 \mathrm{~m}$ people. ${ }^{3}$

The drug cimetidine was chosen for the feasibility study because it was first marketed only a few years ago but rapidly became widely prescribed. The aims of the study were to determine the practicability and the cost of the method and to evaluate the nature of the contribution that could be made to the detection of adverse drug reactions.

\section{Materials and methods}

Photocopies were obtained of all the prescriptions for cimetidine submitted to the prescription pricing division by chemist contractors in the Tayside region over the period August 1980 to April 1981. These would include almost all the prescriptions written by general practitioners that were actually dispensed, although a small but unknown number of patients may have cashed their prescriptions outside the Tayside region. The date of the prescriptions together with the general practitioner reference number (which is printed on all Scottish prescriptions) and the patient community health number (see below) were keyed into the area health board computer. 


\section{PATIENT IDENTIFICATION}

Every patient registered with a Tayside general practitioner has been allocated a unique community health number (CHNo) which is held in the area health board computer together with patient biographical details. ${ }^{2}$ The health board provided an alphabetical listing of these patients on microfiche. The cimetidine scripts were matched to the microfiche listing using the patient's name and address and the general practitioner reference number to obtain the appropriate CHNo.

\section{MORBIDITY DATA}

For every patient discharged from hospital the main medical condition and up to five other conditions and two surgical operations are recorded on the form SMR1 which has been described by Heasman. ${ }^{4}$ Diagnoses are coded to the 9th revision of the International Classification of Disease, ${ }^{5}$ and operations to the OPCS Classification of Surgical Operations. ${ }^{6}$ The data from the SMR1 forms are stored in the area health board computer, and each record may be accessed through the patient's community health number. Discharge details for all cimetidine takers for whom a valid $\mathrm{CHNo}$ was obtained were retrieved by specially developed computer programs for the period from the date of the prescription to 31 December 1981.

\section{CONTROL SELECTION}

A control was obtained for each cimetidine taker matching for age (within five years), sex, and general practitioner. For those cases whose general practitioner reference number was invalid-for instance, owing to retiral or migration of the general practitioner-the control was selected matching only by age and sex. Similarly for those cases in which a control could not be matched within five years of age, the requirement for general practitioner matching was dropped.

\section{Results}

\section{IDENTIFICATION OF PATIENTS}

A total of 12861 prescriptions dispensed in Tayside during the period August 1980 to April 1981 were obtained from the prescription pricing division. A valid community health number was identified for $9754(76 \%)$ of these prescriptions. From these a total of 3802 patients were identified and these had received an average of 2.6 prescriptions each. Of the patients identified, 2201 were male and 1601 were female, indicating that $1.2 \%$ of men and $0.8 \%$ of women in the Tayside region had received cimetidine over a nine month period. The higher frequency among men is highly statistically significant $(\mathrm{p}<0.001)$.
FREQUENCY OF ADMISSION TO HOSPITAL

The computerised hospital medical records were searched for details of all hospital admissions between the date of the prescription and 31 December 1981, an average duration of follow up of 12 months: 929 of the cimetidine takers had at least one hospital admission and in all there were 1613 admissions to hospital. Table 1 shows that just over twice as many cimetidine takers had at least one admission to hospital as control patients matched by age, sex, and general practitioner. When analysed separately, men and women showed similar increased frequencies of hospital admissions.

Table 1 Hospital discharges* among cimetidine takers and controls

\begin{tabular}{lcll}
\hline & Cimetidine & Control & Ratio \\
\hline Persons with one or more discharges: & 929 & 401 & $2 \cdot 3$ \\
Male & 513 & 236 & $2 \cdot 2$ \\
Female & 416 & 165 & $2 \cdot 5$ \\
& & & \\
Total number of discharges: & 1613 & 654 & $2 \cdot 5$ \\
Male & 903 & 388 & $2 \cdot 3$ \\
Female & 710 & 266 & 2.7 \\
\hline
\end{tabular}

*Between the date of issue of the prescription and 31 December 1981.

DISCHARGE DIAGNOSES AMONG CIMETIDINE TAKERS

To compare the morbidity experience of the cimetidine takers and the controls, the total numbers of discharge diagnoses have been presented (table 2). The reasons for using this measure rather than numbers of patients are discussed in appendix 1. As might have been expected the greatest excess morbidity among cimetidine takers was for diseases of the digestive system. But large excesses were also seen in other systems, particularly the musculoskeletal system, neoplasms, and the respiratory system. Smaller excesses were seen with the circulatory and genitourinary system, and no excesses were seen for diseases of the endocrine system, the nervous system, or the skin.

Table 2 Number of discharge diagnoses in major disease groupings

\begin{tabular}{llcc}
\hline & \multicolumn{2}{l}{ No of diagnoses } & \\
\cline { 2 - 4 } Disease group (ICD No*) & Cimetidine & Control & Ratio \\
\hline Digestive system (520-579) & 515 & 52 & 9.9 \\
Signs, symptoms, and ill-defined & & & \\
$\quad$ conditions (780-799) & 195 & 62 & 3.1 \\
Musculoskeletal system (710-739) & 72 & 38 & 1.9 \\
Neoplasms (140-239) & 163 & 87 & 1.9 \\
Respiratory system (460-519) & 93 & 53 & 1.8 \\
Circulatory system (390-459) & 199 & 166 & 1.7 \\
Genitourinary system (580-629) & 89 & 58 & $1 \cdot 5$ \\
Diseases of the skin (680-709) & 30 & 26 & 1.2 \\
Nervous system and sense organs & & & \\
$\quad$ (320-389) & 42 & 39 & 1.1 \\
\hline
\end{tabular}

-Ninth Revision of the International Classification of Diseases. 
Table 3 presents a more detailed analysis of the diseases of the digestive system. The largest excesses were seen for peptic ulcers and oesophagitis, the diseases for which cimetidine is prescribed. Gastrointestinal haemorrhage may also have been in excess because in many cases it will have been a complication of an ulcer. Diseases of the gallbladder and biliary tract are unlikely to be related to ulcers, but they frequently present with symptoms of dyspepsia. $^{7}$

A similar analysis of the category "signs, symptoms, and ill-defined diseases" showed that the excess among cimetidine takers is largely restricted to the abdomen (table 4). These excesses could arise because insufficient information is recorded on the SMR1 to enable a precise diagnosis to be coded. Alternatively, it could be that patients with vague aches and pains in the abdomen are first prescribed cimetidine by their general practitioner and are subsequently referred to hospital.

\section{COSTING OF THE RECORD LINKAGE STUDY}

The cost of a study is an important element of its feasibility assessment, and table 5 shows that for a record linkage these are quite modest. The principal reason for this is that the morbidity data are already collected and computerised by the area health board for other purposes. Thus the clerical and data processing costs are low because they relate only to the processing of the prescriptions. In fact the largest cost is that of the salaries of the senior staff concerned in the design and analysis of the study.

The overall study cost is, however, a preliminary rather than final value, since some further analysis has still to be conducted. If this additional research were substantial or entailed the review of a series of case notes the eventual cost of the analysis could be as much as twice the value of the $£ 6000$ shown.

Table 5 also shows the cost of repeating the study with 10000 patients taking a drug and a similar number taking a control drug. The cost of data collection has been scaled up in proportion to the study size and assumes that the ratios of prescriptions to patients will be similar to that for cimetidine. Nevertheless, since many features of the study design and the computer programs that were developed in the feasibility study could be used in any future exercise, the expected costs of these activities will be lower than for the feasibility study. Thus the cost of the whole study would be around $£ 20000$. These calculations also assume that the staff processing and analysing the data would be paid only for the time for which they are actually required. In addition, they make no allowance for costs such as rent of premises or heating and lighting, and should thus be regarded as minimum estimates.
Table 3 Numbers of discharge diagnoses for diseases of the digestive system

\begin{tabular}{|c|c|c|c|}
\hline \multirow[b]{2}{*}{ Disease group (ICD No*) } & \multicolumn{3}{|c|}{ No of diagnoses } \\
\hline & Cimetidine & Control & Ratio \\
\hline \multicolumn{4}{|c|}{ All digestive system (excluding cancer) } \\
\hline$(520-579)$ & 515 & 52 & 9.9 \\
\hline \multicolumn{4}{|l|}{ Oesophagus, stomach, and duodenum } \\
\hline$(530-537)$ & 331 & 9 & - \\
\hline Peptic ulcers (531-533) & 207 & 2 & - \\
\hline Diseases of the oesophagus (530) & 87 & 3 & - \\
\hline \multicolumn{4}{|l|}{ Other diseases of the stomach and } \\
\hline duodenum (534-537) & 37 & 4 & - \\
\hline Gastrointestinal haemorrhage (578) & 27 & 1 & - \\
\hline $\begin{array}{l}\text { Gallbladder and biliary tract } \\
\text { (574-576) }\end{array}$ & 35 & 2 & - \\
\hline
\end{tabular}

*Ninth Revision of the International Classification of Diseases.

Table 4 Discharge diagnoses for signs, symptoms, and ill-defined conditions

\begin{tabular}{llll}
\hline & \multicolumn{2}{l}{ No of diagnoses } & \\
\cline { 2 - 4 } Disease group (ICD No*) & Cimetidine & Control & Ratio \\
\hline $\begin{array}{c}\text { Symptoms (780-789) } \\
\text { Symptoms affecting digestive system } \\
\quad(787)\end{array}$ & 186 & 62 & 3.0 \\
$\begin{array}{l}\text { Abdominal pain (789.0) } \\
\text { All other symptoms }\end{array}$ & 73 & 2 & - \\
All other signs and ill defined & 79 & 5 & - \\
conditions (790-799) & 9 & 0 & 1.4 \\
\hline
\end{tabular}

*Ninth Revision of the International Classification of Diseases.

Table 5 Costing of the cimetidine record linkage study

\begin{tabular}{lrr}
\hline & \multicolumn{1}{l}{ Cost } \\
\cline { 2 - 3 } & $\begin{array}{l}\text { Actual } \\
(£)\end{array}$ & \multicolumn{1}{c}{$\begin{array}{l}\text { Future } \dagger \\
(£)\end{array}$} \\
\hline Photocopying and transport of the & $1000^{*}$ & 5000 \\
$\begin{array}{l}\text { prescriptions } \\
\text { Allocation of CHNo }\end{array}$ & 2000 & 10000 \\
$\begin{array}{l}\text { Data preparation } \\
\text { Program development and computing }\end{array}$ & $100^{*}$ & 500 \\
$\quad$ costs & $3500^{*}$ & 500 \\
Study design and analysis & 6000 & 4000 \\
Total & $£ 12600$ & $£ 20000$ \\
\hline
\end{tabular}

* No charge was made for these items.

tFor a study of $\mathbf{1 0 0 0 0}$ patients on each of a test and control drugs.

\section{Discussion}

The aim of this study was to assess both the feasibility of record linkage and the contribution that it could make to the detection of adverse drug reactions. The results showed that it was possible to match a large proportion $(76 \%)$ of the prescriptions to Tayside residents and that details of their discharge from hospital could be retrieved from computer files. Although $24 \%$ of the prescriptions were not matched, the proportion of cimetidine takers not 
identified will be lower than this because many patients will have received more than one prescription.

Just under 4000 cimetidine takers were identified, many fewer than the figure of 10000 that other workers have suggested as a suitable target for postmarketing surveillance studies. ${ }^{8}$ The population of Tayside is 400000 so that large patient cohorts could be obtained only for drugs prescribed at high frequencies. Two other Health Boards, however, Grampian and Argyll and Clyde, are currently developing similar computerised systems. The combined population of these three areas would be $1 \cdot 2 \mathrm{~m}^{3}$ and would be sufficient to obtain large sample sizes for drugs prescribed to $1 \%$ of the population. Skegg and Doll suggested that a drug surveillance project should cover a population of at least half a million people, ${ }^{1}$ but clearly a much larger population would be required to cover the less commonly prescribed drugs.

To aid in the interpretation of the morbidity data the cimetidine takers were compared with an equal number of community based controls matched by age, sex, and general practitioner. For the present feasibility study these controls proved informative but, as is discussed below, in further studies of postmarketing surveillance a more suitable comparison group may be required.

Overall, cimetidine takers were twice as likely to enter hospital as the controls. The greatest excess was seen among diseases of the gastrointestinal tract, particularly peptic ulcers and oesophagitis. These excesses would be expected, since cimetidine is prescribed for these diseases, and it is encouraging that the method detected them so clearly. Cimetidine takers also showed large excesses for several other disease categories, most notably diseases of the musculoskeletal, respiratory, and cardiovascular systems, and all cancers. Another postmarketing surveillance study of cimetidine which used different methods, has obtained remarkably similar results. ${ }^{910}$ But probably the associations are indirect rather than casual and explanations can be offered for many of them. Thus both chronic respiratory and cardiovascular diseases are known to be associated with peptic ulcers, ${ }^{11}$ which would lead to the observed association with cimetidine. For respiratory disease it could be suggested that smoking, a known risk factor for peptic ulcers, ${ }^{12}$ was the common link. It has also been suggested that the dyspepsia caused by non-steroidal anti-inflammatory drugs may lead to cimetidine being frequently prescribed for patients with musculoskeletal disease. ${ }^{9}$ The excess of cancers is unlikely to be casual because these diseases are thought to take many years to develop ${ }^{13}{ }^{14}$ and the maximum follow up period in this study was 18 months.

The above explanations are simply specific examples of the more general observations that the morbidity experience of cimetidine takers largely reflects the disease that led to the prescribing of the drug. It is clear that to interpret the morbidity data from drug surveillance studies will require a comparison group which would aid in controlling for these confounding factors. Inman has made the attractive suggestion of identifying patients taking a control drug that is "a chemically or pharmacologically similar drug already marketed for the same indication." 8 This would present no difficulties for the record linkage approach as the same methods of patient identification and follow up could be used for the index and the control drug. Further, it would produce only a small increase in the cost of the study.

Although intended as a test of the feasibility of record linkage, this study has highlighted the problems of distinguishing adverse reactions from among the other morbidity of drug takers. Thus if an adverse reaction with a frequency of one per 1000 drug takers had occurred in the present study we would have expected to see only about four cases of the adverse reaction. The detection of a small number of cases of an adverse reaction, even when an appropriate "similar drug" control group is available, is the major outstanding problem facing all methods of postmarketing drug surveillance. The acid test of all the methods currently being evaluated will be the detection of a previously unsuspected adverse reaction.

The central feature of the record linkage method and the source of its advantages and limitations is the use of morbidity data that are routinely processed and computerised by the area health board. The principal benefit of this is its cheapness: the expensive part of other postmarketing studies is the collection of the morbidity data which for the present study are obtained for the cost of developing and running a computer program. Two published estimates for the cost of a monitored release scheme with 10000 patients were $£ 1.2 \mathrm{~m}$ and $£ 2.0 \mathrm{~m} \cdot{ }^{15}{ }^{16}$ In contrast, a study of similar size carried out by record linkage would cost $£ 20000$.

A further advantage of record linkage is that the follow up period may be extended indefinately, simply by rerunning the computer programs at convenient intervals. Extended follow up may not be possible with other methods of postmarketing surveillance as the cost of repeating the expensive data collection process may be prohibitive. Thus record linkage may be the only practical method of 
detecting adverse reactions which take several years to be expressed.

One limitation of the use of hospital morbidity data is that only serious adverse reactions will be identified. But this restriction will also mean that the volume of morbidity data will be much smaller than if minor illnesses (which would be far greater in number) were included. This will simplify the processing and interpretation of the morbidity data and make it easier to identify small numbers of adverse reactions. The present study, however, could be regarded as complementary to Inman's prescription-event monitored scheme which investigates morbidity in general practice. ${ }^{8}$

A potentially more serious limitation of this system is its reliance on the accuracy of coding of discharge diagnoses. Although a study by Lockwood found the coding accurate for $94 \%$ of the diagnoses,${ }^{17}$ others have claimed that a higher level of error may occur. ${ }^{18-21}$ Certainly the finding among crimetidine takers of large numbers of diagnoses in the category "symptoms, signs, and ill-defined conditions" suggests that there may be a problem. More accurate diagnoses could be obtained from a review of the case notes, but because of the cost this would only be possible for a limited number of patients. These would be identified from a detailed analysis of the morbidity data which would indicate particular disease groups for further study. In the longer term the screening of the data could be computerised, with a signal being generated automatically whenever an arbitrary level of excess morbidity was reached.

The conclusion of this study is that record linkage in Tayside can provide a simple and inexpensive method of postmarketing drug surveillance. It is particularly suited for the detection of serious adverse reactions which may take several years to develop.

We gratefully acknowledge the contribution of the late Professor James Crooks whose inspiration and enthusiasm made this study possible. We also thank the Prescription Pricing Division (Scotland) for providing photocopies of prescriptions and the Tayside Health Board Computer Consortium and $\mathrm{Mr}$ J H Scott and Mr D Butterworth for providing access to the computerised data and developing the linkage programs. This study was supported by a grant from the Department of Health and Social Security and the Scottish Home and Health Department.
Appendix

NUMBERS OF PATIENTS AND NUMBERS OF DISCHARGE DIAGNOSES

When a group of patients is followed up through time many will have several repeat admissions for the same illness and some will have separate admissions for different diseases. To obtain the number of patients with diseases in a particular category each patient should be counted only once so that repeat admissions do not inflate the figures. But when we are dealing with a broad disease category a patient may have had more than one disease in the same category but would be counted only once. For example, a patient with separate admissions for appendicitis and cirrhosis of the liver would count only once within the category diseases of the digestive system. Clearly, instead of counting the number of patients in a category we should count the number of diseases. This, however, cannot be obtained without a detailed review of all the patient's case notes and careful definition of what constitutes a separate disease. As an alternative the total number of discharges may be used, making the assumption that for each episode of an illness cimetidine takers and controls have the same average number of discharge diagnoses. Although this assumption will not hold if cimetidine takers experience more or fewer repeat admissions, as a single measure it may be more informative than patient numbers. In practice, both measures would be used, and any disease category which either of them signalled as being of potential interest would be investigated further.

\section{References}

${ }^{1}$ Skegg DCG, Doll R. Record linkage for drug monitoring. J Epidemiol Community Health 1981; 35: 25-31.

${ }^{2}$ Angus J, Cameron L, Finlayson CF, Hopkins RT, Martin $\mathrm{KB}$, Scott JH. The Tayside master patient index. Dundee: Tayside Health Board, 1978.

${ }^{3}$ Registrar General Scotland. Annual report 1981. Edinburgh: HMSO, 1982: 26.

- Heasman MA. Scottish hospital in-patient statistics-sources and uses. Health Bull (Edinb) 1968; 26: $10-8$.

${ }^{5}$ World Health Organisation. International classification of diseases. Vol 1. London: HMSO, 1977.

${ }^{6}$ Office of Population Censuses and Surveys. Classification of surgical operations. 3rd rev. London: OPCS, 1975.

${ }^{7}$ Horrocks JC, de Dombal FT. Clinical presentation of patients with dyspepsia. Gut 1978; 19: 19-26.

${ }^{8}$ Inman WHW. Postmarketing surveillance of adverse drug reactions in general practice. $\mathrm{Br}$ Med $J$ 1981; 282: 1131-2; 282: 1216-7. 
${ }^{9}$ Colin-Jones D, Langman MJS, Lawson DH, Vessey MP. Postmarketing surveillance. In: Baron $\mathrm{JH}$, ed. Cimetidine in the 80s. Edinburgh: Churchill Livingstone, 1981: 270-4.

${ }^{10}$ Colin-Jones DG, Langman MJS, Lawson DH, Vessey MP. Postmarketing surveillance of the safety of cimetidine: 12 month mortality report. $\mathrm{Br} \mathrm{Med} J \mathrm{1983}$; 286: 1713-6.

${ }^{11}$ Langman MJS, Cooke AR. Gastric and duodenal ulcers and their associated diseases. Lancet 1976; i: 680-3.

${ }^{12}$ Bouchier IAD. Peptic ulcer: the changing scene. Scott Med J 1981; 26: 297-302.

${ }^{13}$ Cole P, Goldman MB. Occupation. In: Fraumeni JF, ed. Persons at high risk of cancer. New York: Academic Press, 1975: 167-84.

${ }^{14}$ Hoover R, Fraumeni JF. Drugs. In: Fraumeni JF, ed. Persons at high risk of cancer. New York: Academic Press, 1975: 185-99.

${ }^{15}$ Godfrey D, Bowler EJ. Postmarketing surveillance-the commercial implications. In: Postmarketing surveillance of adverse reactions to new medicines. (Publication No 7.) London: Medico-Pharmaceutical Forum, 1977: 27-39.
${ }^{16}$ Sullman SF. A resumé of the pharmaceutical industry's experiences with monitored release. In: Postmarketing surveillance of adverse reactions to new medicines. (Publication No 7.) London: Medico-Pharmaceutical Forum, 1977: 12-8.

${ }^{17}$ Lockwood E. Accuracy of Scottish hospital morbidity data. Br J Prev Soc Med 1971; 25: 76-83.

${ }^{18}$ Patel AR, Gray G, Lang GD, Baillie FGH, Fleming L, Wilson GM. Scottish hospital morbidity data-1. Errors in diagnostic returns. Health Bull (Edinb) 1976; 34: 215-20.

${ }^{19}$ Patel AR, Gray G, Lang GD, Baillie FGH, Fleming L, Wilson GM. Scottish hospital morbidity data-2. An attempt to improve the standard of diagnostic entries and records in a medical unit. Health Bull (Edinb) 1976; 34: 221-7.

${ }^{20}$ Patel AR, Gray G, Lang GD, Baillie FGH, Fleming L, Wilson GM. Scottish hospital morbidity data-3. Some suggestions for improvement of the accuracy. Health Bull (Edinb) 1976; 34: 227-8.

${ }^{21}$ Lennox B, Clarke JA, Drake F, Ewen SWB. The incidence of salivary gland tumours in Scotland: accuracy of national records. Br Med J 1978; i: 687-9. 